\title{
GaN-based VCSELs: Analysis of internal device physics and performance limitations
}

\author{
Joachim Piprek ${ }^{\mathrm{a}}$ and Simon $\mathrm{Li}^{\mathrm{b}}$ \\ ${ }^{a}$ NUSOD Institute LLC, Newark, DE 19714-7204, United States, E-mail: piprek@ieee.org \\ ${ }^{\mathrm{b}}$ Crosslight Software Inc., 121-3989 Henning Drive, Burnaby, BC V5C 6P8 Canada
}

\begin{abstract}
GaN-based vertical-cavity surface-emitting lasers (VCSELs) are expected to exhibit several advantages over their already widely used edge-emitting counterparts, including lower manufacturing costs, circular output beams, and longer lifetime. However, in contrast to the great success of GaN-based edge-emitting lasers in recent years, GaN-VCSELs still face significant challenges. Electrically pumped devices have been demonstrated only recently and they exhibit severe performance restrictions. We here analyze these recently manufactured GaN-VCSELs using advanced laser simulation software. The simulation self-consistently combines carrier transport, photon emission, and multi-mode optical wave guiding. For the quantum wells, Schrödinger and Poisson equations are solved iteratively at every bias point to account for the Quantum-Confined Stark Effect. Our analysis shows that thick quantum wells allow for the almost complete elimination of the built-in quantum well polarization field. The simulations also reveal several performance limiting effects, e.g., gain-peak offset, current crowding, and electron leakage. Design optimization options are discussed and simulated.
\end{abstract}

Keywords: GaN-based laser, vertical-cavity surface-emitting laser, VCSEL, numerical simulation, built-in polarization, current crowding, leakage current, electron stopper layer

\section{INTRODUCTION}

GaN-based vertical-cavity surface-emitting lasers (VCSELs) are expected to show several advantages over edgeemitting GaN-lasers, including lower manufacturing costs, circular output beams, and longer lifetime. In contrast to the great success of GaN-based edge-emitting lasers in recent years, GaN-VCSELs still face significant challenges. Electrically pumped GaN-VCSELs have been demonstrated only recently and they exhibit severe performance restrictions. ${ }^{1,2}$

Based on our previous experience with GaN-based VCSEL design and simulation, ${ }^{3}$ we here analyze some recently manufactured GaN-VCSELs using advanced laser simulation software. ${ }^{4}$ The simulation self-consistently combines carrier transport, photon emission, and multimode optical wave guiding. A similar model was previously used to study GaN-based edge-emitting lasers, resulting in excellent agreement between measurements and simulations. ${ }^{5}$ However, self-heating is neglected here as we investigate pulsed operation at room temperature. More details on theoretical models and material parameters are given in the next section. Section 3 and 4 then analyze the design of two recently demonstrated electrically pumped GaN-VCSELs, the first one employing ten $2.5 \mathrm{~nm}$ thin quantum wells and the second one featuring only two 9 nm thick quantum wells .

\section{MODELS AND MATERIAL PARAMETERS}

As the VCSELs exhibit cylinder symmetry, two-dimensional (2D) device simulation with the vertical coordinate $\mathrm{Z}$ and the lateral radius $\mathrm{R}$ is chosen. Our software PICS3D ${ }^{4}$ self-consistently combines the computation of semiconductor transport equations, quantum well bandstructure, optical gain, and optical mode. The transport model includes drift and diffusion of electrons and holes, Fermi statistics, built-in polarization and thermionic emission at hetero-interfaces, as well as spontaneous and defect-related Shockley-Read-Hall (SRH) recombination of carriers. For the quantum wells,

Gallium Nitride Materials and Devices V, edited by Jen-Inn Chyi, Yasushi Nanishi, Hadis Morkoç

Cole W. Litton, Joachim Piprek, Euijoon Yoon, Proc. of SPIE Vol. 7602, 760217 • (c) 2010 SPIE

CCC code: $0277-786 \mathrm{X} / 10 / \$ 18 \cdot$ doi: $10.1117 / 12.0000000$

Proc. of SPIE Vol. $7602760217-1$ 
Schrödinger and Poisson equations are solved iteratively to account for the quantum well deformation with changing device bias. Stimulated emission of photons within the quantum well is calculated by a free carrier model including the wurtzite energy band structure.

An important issue in any device simulation is the selection of appropriate values for the various material parameters. Published values for many parameters of GaN-based compounds vary substantially in the literature. We here mainly employ the nitride parameters given in Refs. 6 and 7. However, the crucial issue of strong self-polarization observed in nitride compounds should be addressed in more detail.

Built-in interface charges due to spontaneous and piezoelectric polarization are known to influence the performance of nitride devices. ${ }^{8}$ Much theoretical and experimental research has been committed to the unusual built-in polarization strength in GaN-based alloys. Figure 1 shows the theoretically predicted interface charge density and the corresponding built-in field as function of alloy composition. ${ }^{9}$ The strong built-in field leads to a separation of electrons and holes within the quantum well and thereby to a reduction of the photon emission rate. However, experimental investigations of similar quantum wells often give weaker built-in fields than predicted, ranging from $20 \%{ }^{10}$ to $80 \%^{11}$ of the theoretical value, with typical results near $50 \% .^{12}$ This broad variation has been attributed to partial compensation of the polarization field by fixed defect and interface charges ${ }^{13}$ or to inappropriate analysis of measured data. ${ }^{14}$ In the following, we assume only $50 \%$ of the theoretically predicted polarization charges.

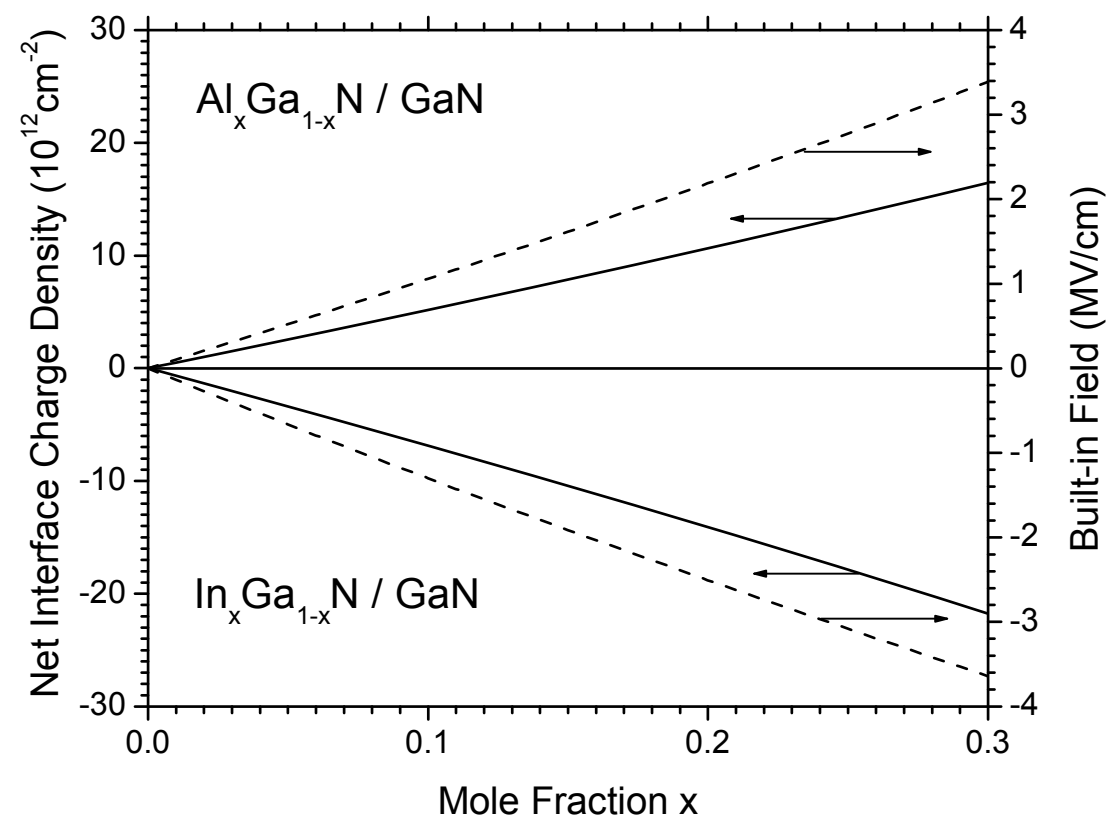

Figure 1: Polarization charges and corresponding built-in field at InGaN/GaN and AlGaN/GaN interfaces. ${ }^{9}$

\section{VCSEL WITH TEN THIN QUANTUM WELLS}

The first design investigated is tailored for light emission at $\lambda=460 \mathrm{~nm} .{ }^{1}$ The 5 - $\lambda$-cavity is sandwiched between a 29 period AlN/GaN distributed Bragg reflector (DBR) on the n-doped side and an 8-period $\mathrm{Ta}_{2} \mathrm{O}_{5} / \mathrm{SiO}_{2}$ dielectric DBR on the p-side (Fig. 2). The multi-quantum well (MQW) active region comprises $10 \mathrm{InGaN}$ quantum wells (each $2.5 \mathrm{~nm}$ thick) that are separated by $7.5 \mathrm{~nm}$ GaN barriers. The current is injected through a $240 \mathrm{~nm}$ thick indium tin oxide (ITO) layer on the $\mathrm{p}$-side ( $5 \mu \mathrm{m}$ aperture radius) and an intra-cavity ring contact on the $\mathrm{n}$-side. This VCSEL shows continuouswave $(\mathrm{CW})$ lasing only at very low temperatures $(77 \mathrm{~K})$. One reason for this is the significant optical loss caused by the thick ITO layer $(1.4 \%)$. This loss can be reduced by using a thinner ITO layer that is placed at the node of the standing wave. 
As both the cavity wavelength and the QW gain spectrum red-shift with rising temperature, a careful adjustment of the QW gain spectrum is required to achieve lasing at room temperature. ${ }^{15}$ Our simulation adjusts the QW composition to move the room-temperature gain peak into close proximity of the cavity wavelength (Figs. 3, 4). However, the MQW active region is wider than the peak of the standing optical wave and some of the quantum wells do not contribute to the lasing process (Fig. 5). The calculated gain enhancement factor is 1.0. On the other hand, this thick MQW region makes the VCSEL less sensitive to growth variations which result in a vertical shift of the optical intensity peak relative to the MQW.

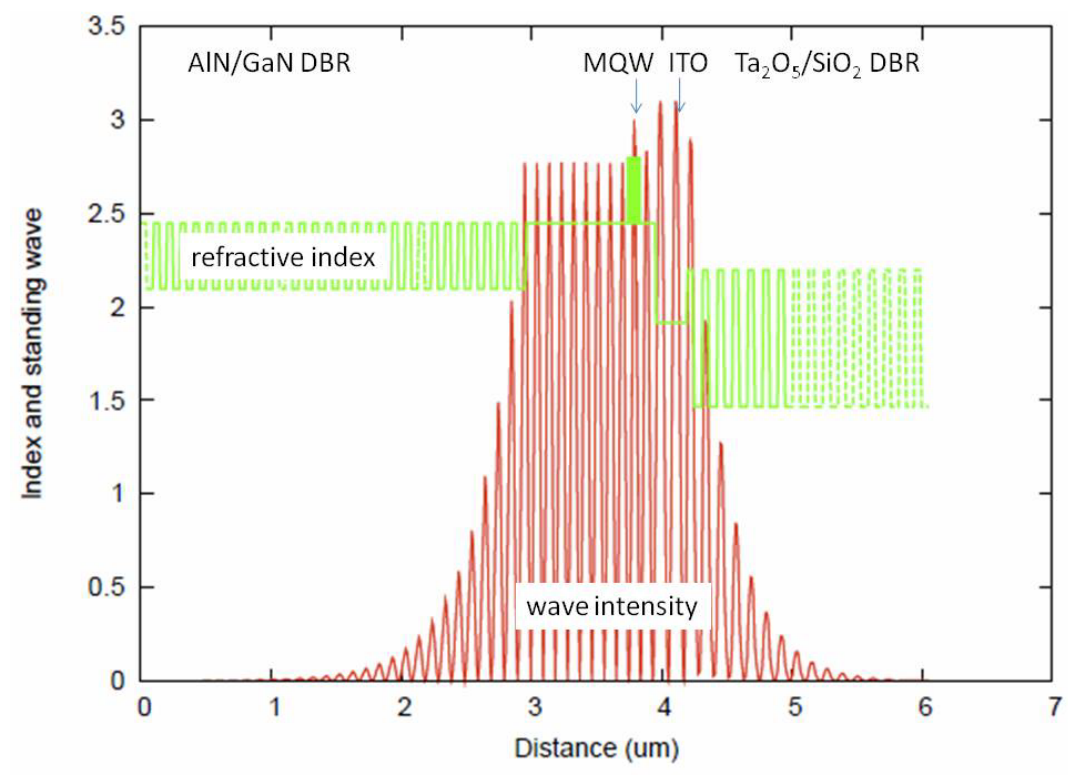

Figure 2. Vertical index profile and optical intensity of the 460nm VCSEL.

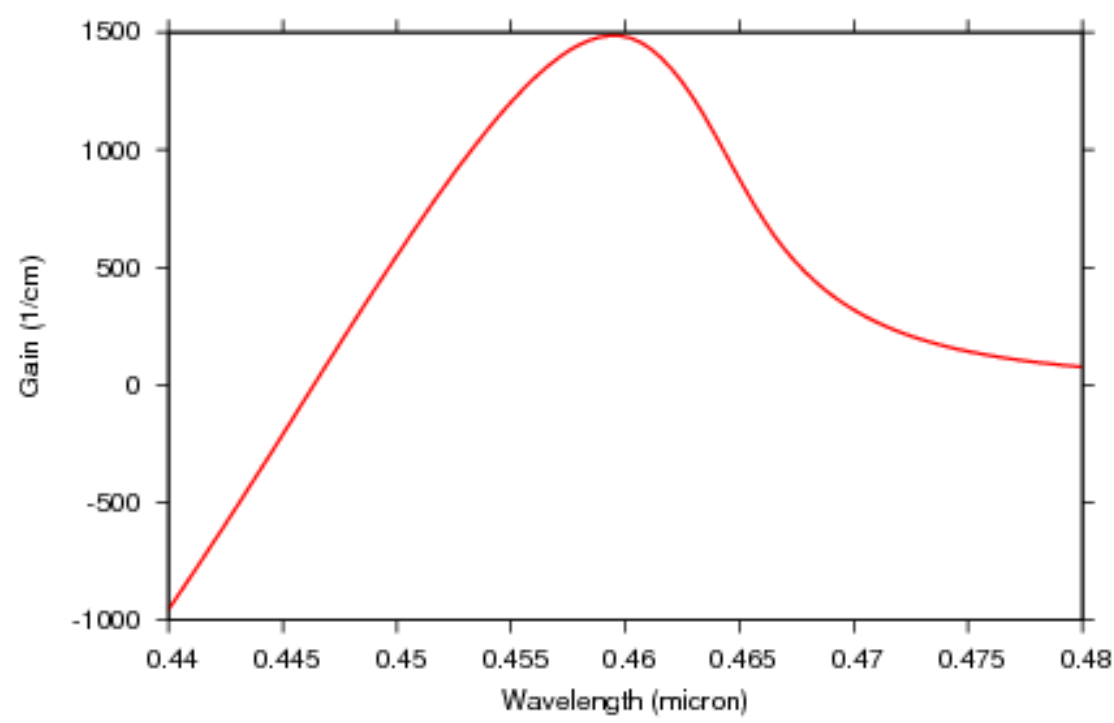

Figure 3. Quantum well material gain spectrum at $\mathrm{T}=300 \mathrm{~K}$. 


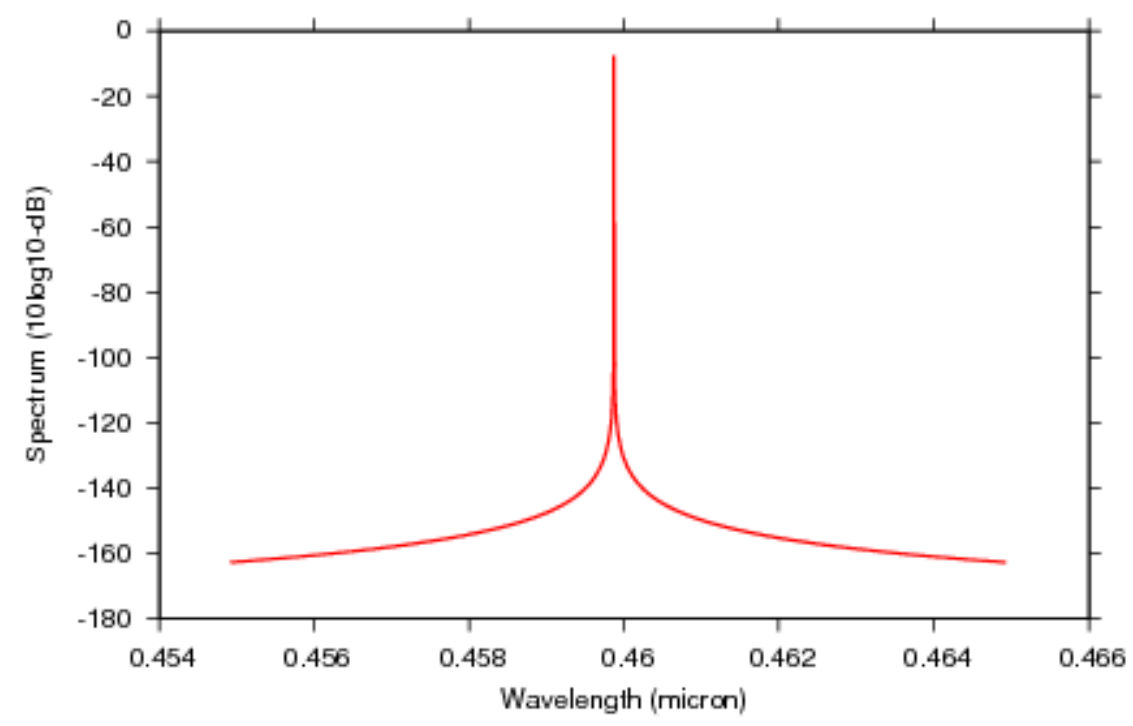

Figure 4. Lasing mode spectrum at $\mathrm{T}=300 \mathrm{~K}$.

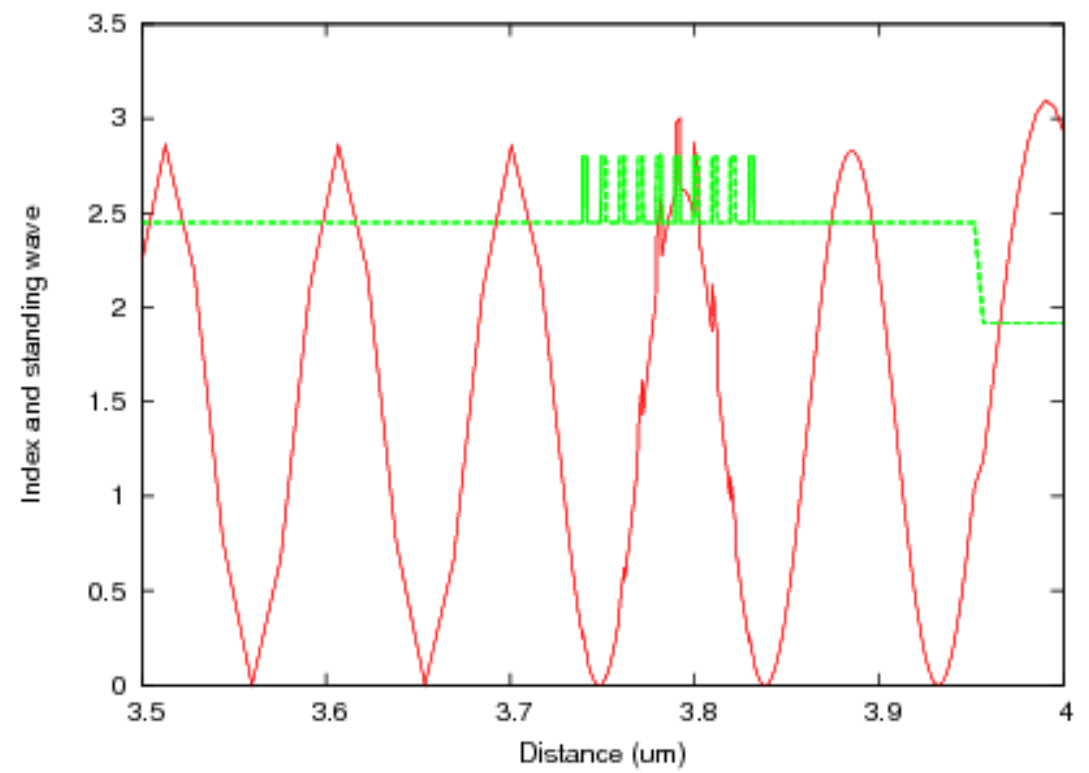

Figure 5. Index and standing wave from Fig. 2 near the MQW.

The calculated electron current profile within the p-GaN layer is plotted in Fig. 6. The sharp peak of the electron current at the ITO aperture radius $(\mathrm{r}=5 \mu \mathrm{m})$ not only reveals significant electron leakage into the $\mathrm{p}$-GaN layer, but also strong current crowding. The electron leakage severely limits the quantum efficiency of the laser. Electron overflow can be reduced by inserting an $\mathrm{AlGaN}$ electron stopper layer above the MQW as it is common with GaN-based edge-emitting lasers. ${ }^{5}$ Such AlGaN layer also reduces the current crowding as it enforces a more uniform hole injection into the MQW. ${ }^{3}$ 


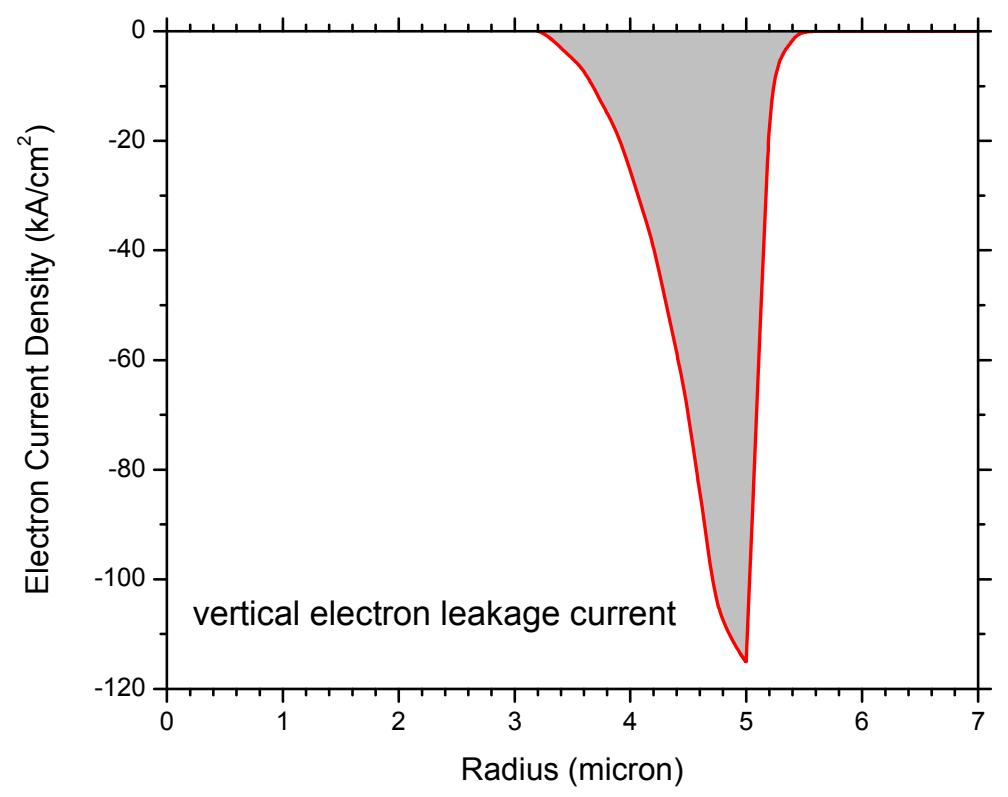

Figure 6. Lateral electron current profile within the $\mathrm{p}-\mathrm{GaN}$ layer above the MQW calculated at $25 \mathrm{~mA}$ injection current (the electron current density is negative in the simulation)

\section{VCSEL WITH TWO THICK QUANTUM WELLS}

The second design investigated is tailored for light emission near $\lambda=410 \mathrm{~nm}^{2}{ }^{2}$ The $7-\lambda$-cavity is sandwiched between an 11.5-period $\mathrm{Nb}_{2} \mathrm{O}_{5} / \mathrm{SiO}_{2}$ DBR on the p-side and a 7-period $\mathrm{Nb}_{2} \mathrm{O}_{5} / \mathrm{SiO}_{2}$ DBR on the n-side (Fig. 7). The active region comprises two $9 \mathrm{~nm}$ thick InGaN quantum wells separated by a $13 \mathrm{~nm} \mathrm{GaN}$ barrier. The current is injected through a $50 \mathrm{~nm}$ thick ITO layer on the p-side $(4 \mu \mathrm{m}$ aperture radius) and an intra-cavity ring contact on the n-side. This GaNVCSEL is the first to show room-temperature continuous-wave (CW) lasing.

The optical simulation results in Fig. 7 show that the ITO layer is well positioned near the node of the standing wave, thereby reducing the optical loss. The two quantum wells are near the peak of the standing wave with a gain enhancement factor of 1.6. As the total thickness of the active region is only $31 \mathrm{~nm}$, precise growth control is more important than in the previous case, as the peak of the standing wave needs to be positioned at the quantum wells (cf. Fig. 5). The strong increase in QW thickness leads to multiple quantum levels that are occupied under lasing operation (Fig. 8). The total carrier distribution is almost uniform inside the quantum wells (Fig. 9) and it is large enough to completely screen the built-in polarization charges, resulting in almost flat-band conditions (Fig. 8). In this case, the built-in QW interface charge density is $5.7 \times 10^{12} \mathrm{~cm}^{-2}$ which requires a QW carrier density of $6.3 \times 10^{18} \mathrm{~cm}^{-3}$ for compensation. As the actual threshold carrier density is more than ten times higher (Fig. 9), polarization screening is achieved within less than one tenth of the QW thickness. This polarization screening in thick quantum wells overcomes the detrimental effect of built-in polarization observed with thin quantum wells. ${ }^{3}$ 


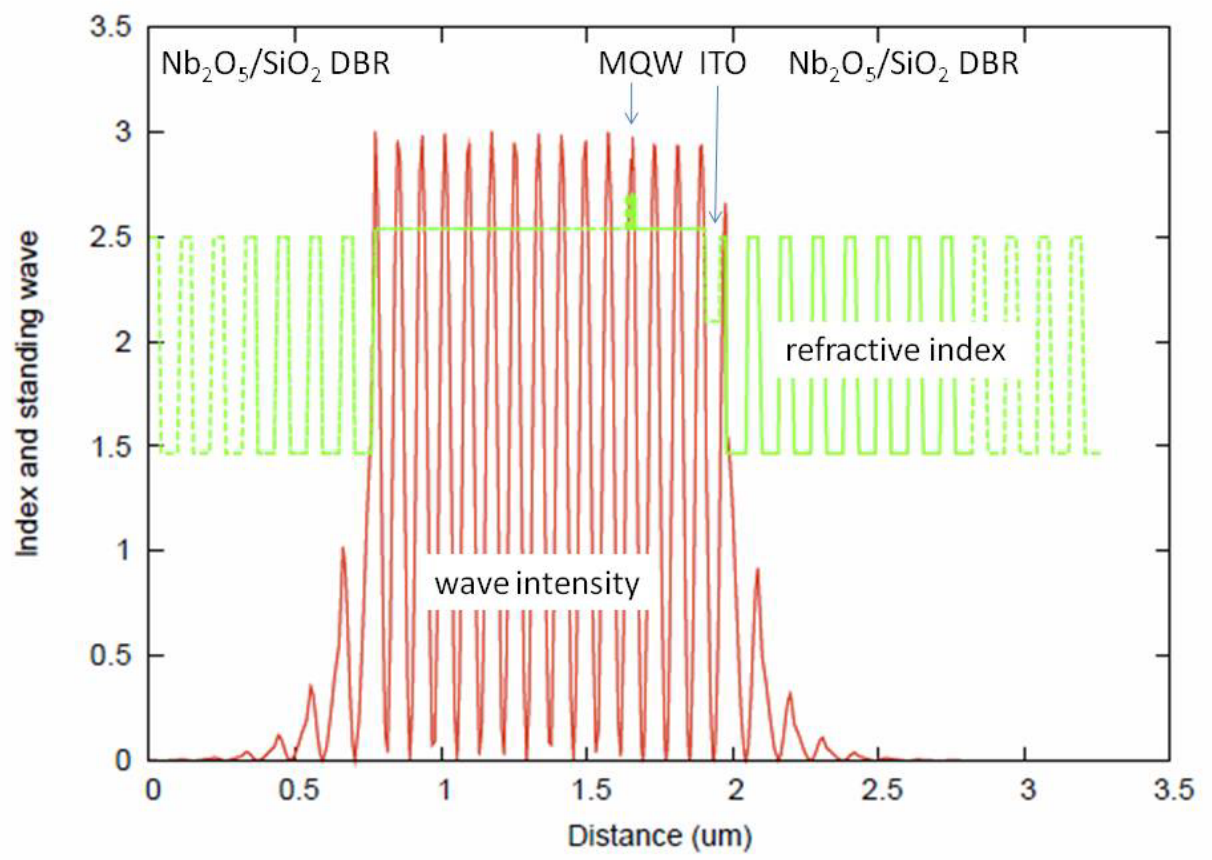

Figure 7. Vertical index profile and optical intensity of the 410nm VCSEL.

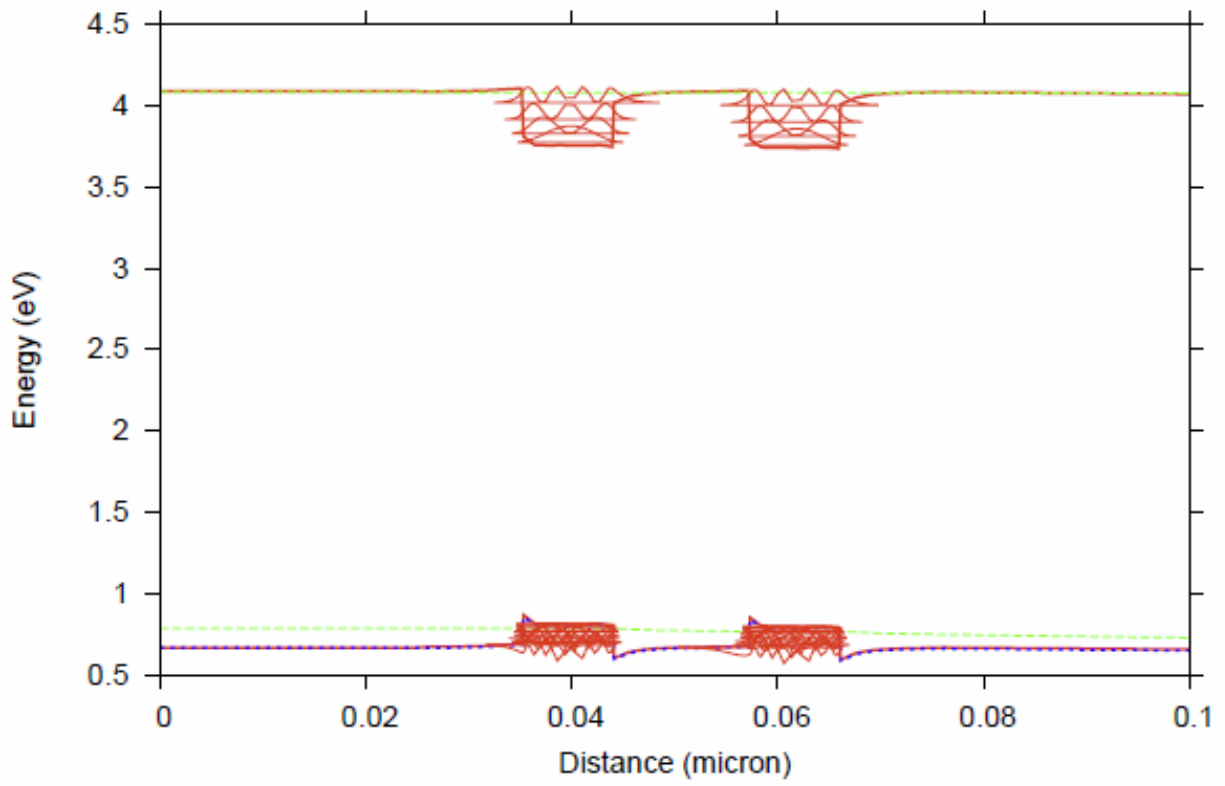

Figure 8. Energy band diagram of the two quantum wells above lasing threshold including quantum levels and wave functions (green dashed lines - quasi Fermi levels). 


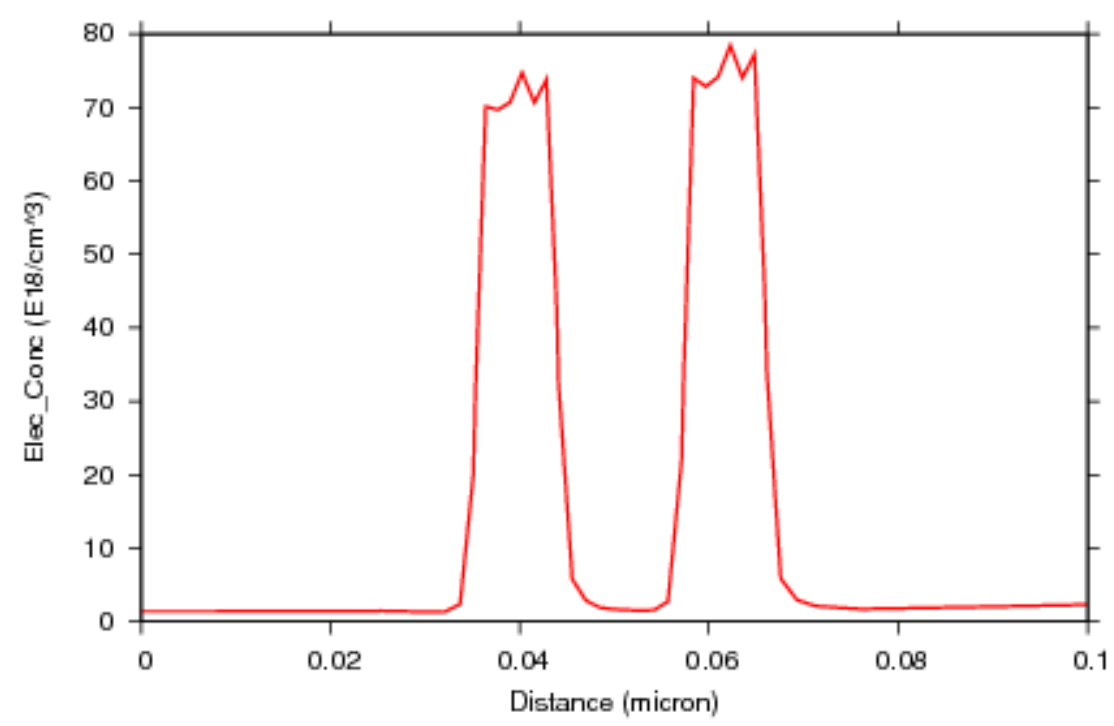

Figure 9. Total electron density on all energy levels inside quantum wells of Fig. 8.

However, also for this improved VCSEL design, our simulations reveal current crowding at the ITO aperture. The resulting gain profile within the quantum wells is non-uniform (Fig. 10) and it mainly supports higher-order optical modes. The top (p-side) quantum well shows a more uniform carrier and gain distribution. We also discover strong electron leakage into the p-GaN layer (Fig. 11). This situation is similar to the previous case (cf. Fig. 6) as both VCSEL designs do not include an $\mathrm{AlGaN}$ electron stopper layer on the $\mathrm{p}$-side of the quantum wells.

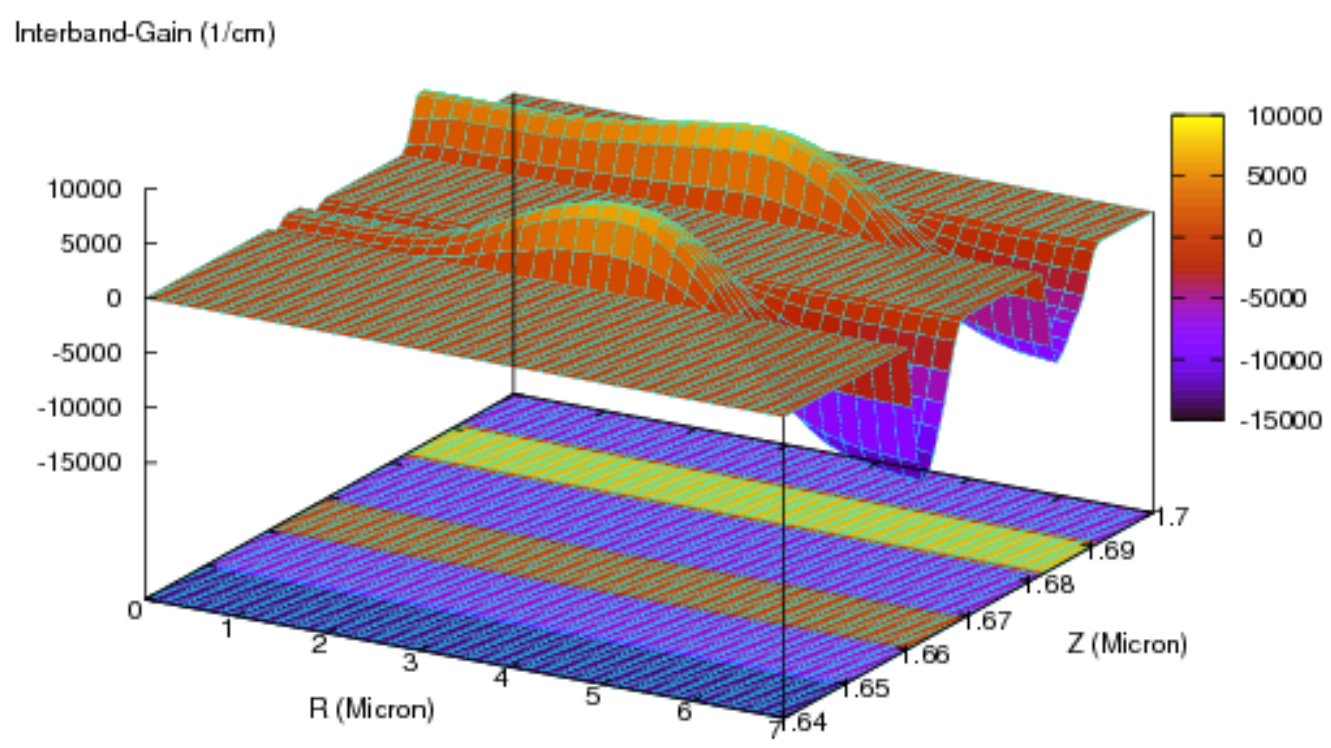

Figure 10. 2D surface plot of the quantum well gain at $12 \mathrm{~mA}$ injection current. 


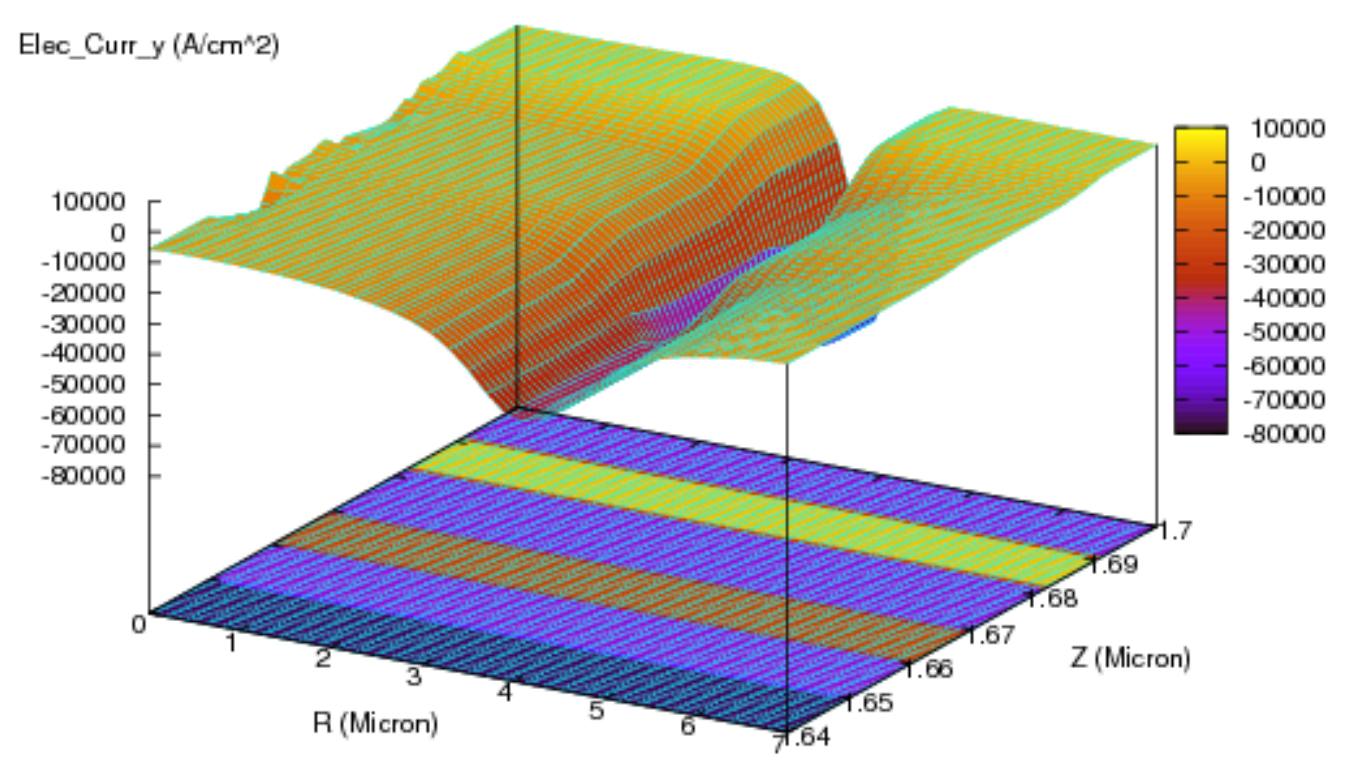

Figure 11. 2D surface plot of the vertical electron current across the QW region at 12mA injection current (cf. Fig. 10).

Figure 12 shows the energy band diagram after inclusion of an $\mathrm{Al}_{0.2} \mathrm{Ga}_{0.8} \mathrm{~N}$ stopper layer above the active region (cf. Fig. 8). The AlGaN interface polarization charges lead to a partial reduction of the energy barrier in the conduction band, however, the resulting light-current characteristic is substantially improved (Fig. 13).

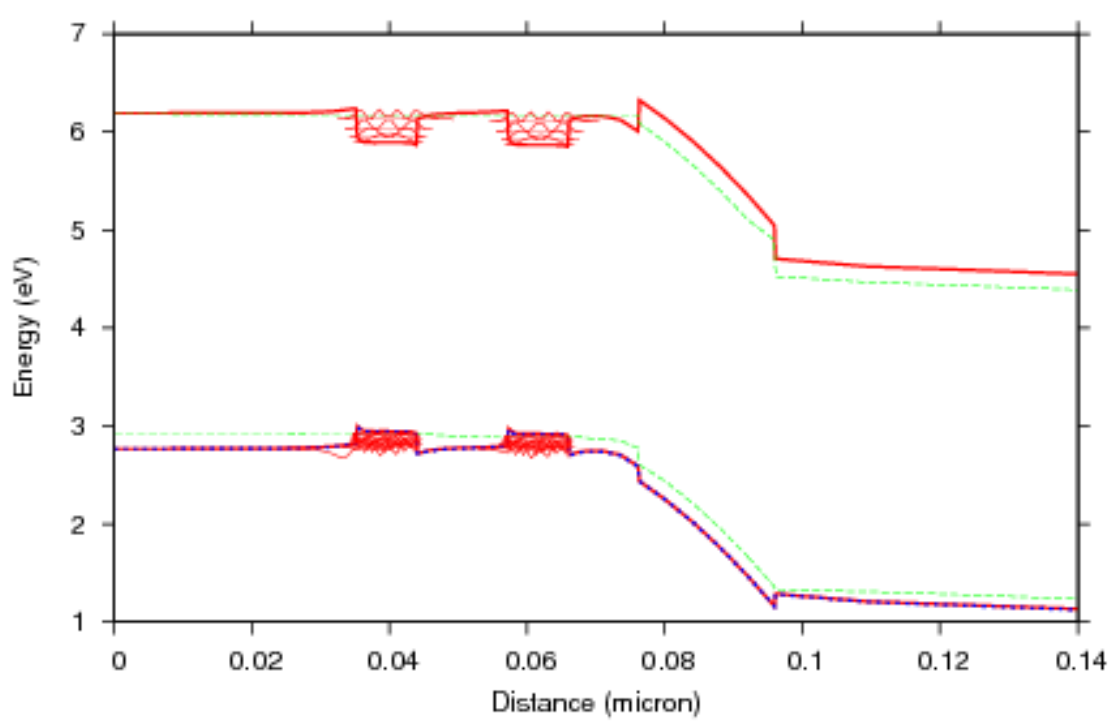

Figure 12. Energy band diagram of InGaN quantum wells and AlGaN electron stopper layer above threshold (including QW wave functions, green dashed lines are the quasi Fermi levels). 


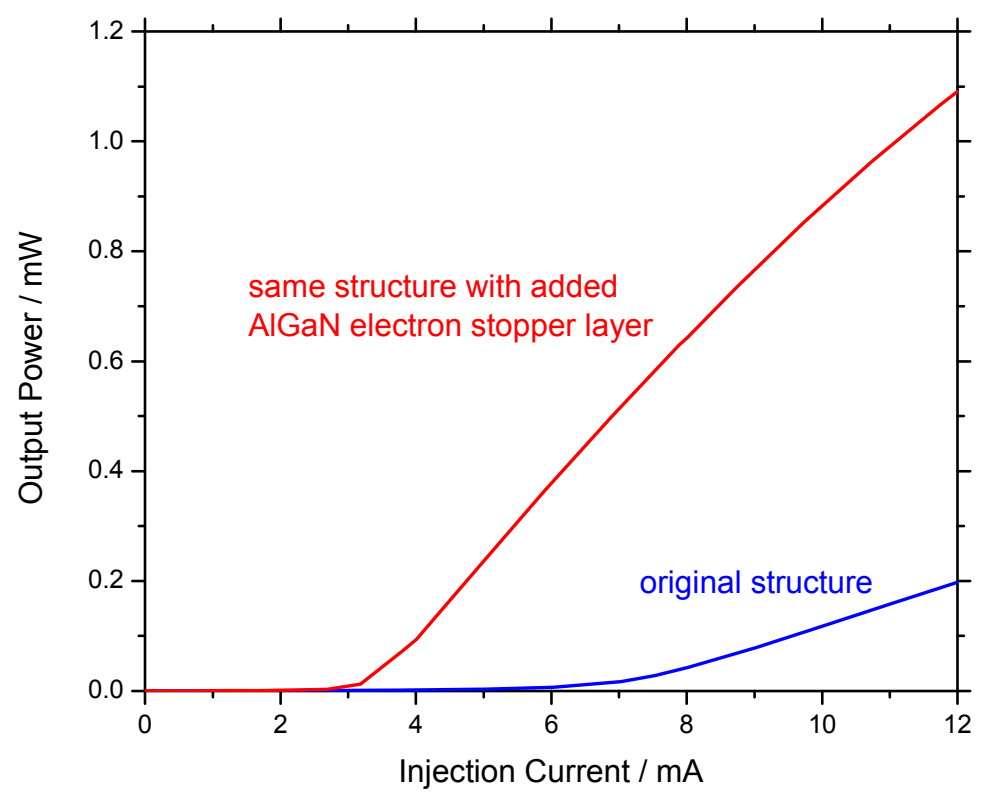

Figure 13. Light-current characteristics simulated for the original 2-QW structure and for the same structure with added AlGaN electron stopper layer.

\section{SUMMARY}

Our simulations reveal the internal device physics of current-injected GaN-VCSEL structures and allow for a detailed design analysis. The first demonstration of room-temperature continuous-wave GaN-VCSEL operation by Nichia ${ }^{2}$ is partially attributed to the use of $9 \mathrm{~nm}$ thick quantum wells which allow for the elimination of the built-in quantum well polarization field. However, our analysis also reveals several performance limiting effects, e.g., current crowding and electron leakage. We demonstrate that electron overflow and threshold current can be reduced substantially by inserting an $\mathrm{AlGaN}$ electron stopper layer above the active region.

\section{REFERENCES}

${ }^{1}$ Tien-Chang Lu, Chih-Chiang Kao, Hao-Chung Kuo, Gen-Sheng Huang, and Shing-Chung Wang, "CW lasing of current injection blue GaN-based vertical cavity surface emitting laser, “Appl. Phys. Lett. Vol. 92, 141102 (2008).

${ }^{2}$ Yu Higuchi, Kunimichi Omae, Hiroaki Matsumura, and Takashi Mukai, "Room-Temperature CW Lasing of a GaNBased Vertical-Cavity Surface-Emitting Laser by Current Injection, “ Appl. Phys. Expr. Vol. 1, 121102 (2008).

3 J. Piprek, Z.-M. Li, R. Farrell, S. P. DenBaars, and S. Nakamura, "Electronic Properties of InGaN/GaN Vertical-Cavity Lasers," Ch. 19 in Nitride Semiconductor Devices- Principles and Simulation, ed. J. Piprek, Wiley-VCH, Weinheim 2007.

${ }^{4}$ PICS3D by Crosslight Software, 2009 (www.crosslight.com).

5 J. Piprek and S. Nakamura, "Physics of high-power InGaN/GaN lasers," IEE Proc.-Optoelectron., vol. 149, p. 145, 2002. 
${ }^{6}$ J. Piprek, Semiconductor Optoelectronic Devices: Introduction to Physics and Simulation, Academic Press, San Diego, 2003.

${ }^{7}$ J. Piprek, H. Wenzel, M. Kneissl, ,Analysis of Wavelength-Dependent Performance Variations of GaN-Based Ultraviolet Lasers", Proc. SPIE 6766, 67660H (2007).

${ }^{8}$ J. Piprek, "Simulation of GaN-based Light Emitting Devices," in: Simulation of Semiconductor Processes and Devices (ed. G. Wachutka and G. Schrag), Springer Verlag, Wien, 2004.

${ }^{9}$ V. Fiorentini, F. Bernardini, and O. Ambacher, "Evidence for nonlinear macroscopic polarization in III-V nitride alloy heterostructures," Appl. Phys. Lett., vol. 80, pp. 1204-1206, 2002.

${ }^{10}$ S. F. Chichibu, A. C. Abare, M. S. Minsky, S. Keller, S. B. Fleischer, J. E. Bowers, E. Hu, U. K. Mishra, L. A. Coldren, and S. P. DenBaars, and T. Sota, "Effective band gap inhomogeneity and piezoelectric field in InGaN/GaN multiquantum well structures,” Appl. Phys. Lett., vol. 73, pp. 2006-2008, 1998.

${ }^{11}$ F. Renner, P. Kiesel, G. H. Döhler, M. Kneissl, C. G. Van de Walle, and N. M. Johnson, "Quantitative analysis of the polarization fields and absorption changes in InGaN/GaN quantum wells with electroabsorption spectroscopy," Appl. Phys. Lett., vol. 81, pp. 490-492, 2002.

${ }^{12}$ H. Zhang, E. J. Miller, E. T. Yu, C. Poblenz and J. S. Speck, "Measurement of polarization charge and conductionband offset at InGaN/GaN heterojunction interfaces," Appl. Phys. Lett., vol. 84, pp. 4644-4646, 2004.

${ }^{13}$ J. P. Ibbetson, P. T. Fini, K. D. Ness, S. P. DenBaars, J. S. Speck, and U. K. Mishra, "Polarization effects, surface states, and the source of electrons in $\mathrm{AlGaN} / \mathrm{GaN}$ heterostructure field effect transistors," Appl. Phys. Lett., vol. 77, $250(2000)$.

${ }^{14}$ I. H. Brown, I. A. Pope, P. M. Smowton, P. Blood, J. D. Thomson, W. W. Chow, D. P. Bour, and M. Kneissl, "Determination of the piezoelectric field in InGaN quantum wells," Appl. Phys. Lett., vol. 86, 131108, 2005.

${ }^{15}$ S. Rapp, J. Piprek, K. Streubel, J. Andre, and J. Wallin, "Temperature sensitivity of $1.54 \mu \mathrm{m}$ Vertical-Cavity Lasers with an InP-based Bragg Reflector,” J. Quantum Electronics, vol. 33, p. 1839 (1997). 